\title{
Fabrication Technologies for Vertically Coupled Microring Resonator With Multilevel Crossing Busline and Ultracompact-Ring Radius
}

\author{
Yasuo Kokubun, Member, IEEE, Yutaka Hatakeyama, Masashi Ogata, Shuichi Suzuki, and Nobuhiro Zaizen
}

\begin{abstract}
To eliminate the scattering loss at the crossing points of cross-grid busline waveguides, the multilevel crossing structure of the busline waveguides was introduced into a vertically coupled microring resonator (VCMRR) filter. To achieve this structure, two fabrication technologies were newly developed; one is a method to planarize perfectly the top surface of each buried waveguide, and the other is a method to fabricate microring waveguides with very smooth sidewalls. Using the latter method, an ultracompact VCMRR with a ring radius of $5 \mu \mathrm{m}$ was realized and a free spectral range of $37 \mathrm{~nm}$ was successfully demonstrated. Next, using the former method, single-ring and quadruple series-coupled ring resonators with multilevel crossing busline waveguides were fabricated. A clear filter response was obtained for the single-microring resonator, and a boxlike filter response was obtained for the quadruple series-coupled microring resonator with multilevel crossing busline waveguides.
\end{abstract}

Index Terms-High-index-contrast optical waveguides, integrated optics, microresonators, optical-waveguide filters.

\section{INTRODUCTION}

$\mathbf{M}$ ICRORING resonator devices are attractive as add-drop wavelength filters for the wavelength division access network, due to their functionality [1], [2] and compactness [3], [4]. We have proposed and demonstrated a vertically coupled microring resonator (VCMRR) filter as the building block of an integrated photonic filter circuit. An ultracompact ring resonator can be realized using a high-index-contrast $(\Delta=34 \%-37 \%)$ waveguide consisting of a glass core $\left(\mathrm{Ta}_{2} \mathrm{O}_{5}-\mathrm{SiO}_{2}\right.$ compound glass with $n=1.78$, or $\mathrm{SiN}$ with $n=2.0)$ and air cladding, and dense integration is possible

Manuscript received July 12, 2004; revised November 10, 2004. This work was supported in part by the Ministry of Education, Culture, Sports, Science and Technology under a Grant-in-Aid for Scientific Research on Priority Areas 13026210, in part by the Futaba Electronics Memorial Foundation, and in part by the 21st Century COE Program of the Ministry of Education, Culture, Sports, Science and Technology.

Y. Kokubun and N. Zaizen are with the Department of Electrical and Computer Engineering, Graduate School of Engineering, Yokohama National University, Yokohama 240-8501, Japan (e-mail: kokubun@ynu.ac.jp).

Y. Hatakeyama was with the Department of Electrical and Computer Engineering, Graduate School of Engineering, Yokohama National University, Yokohama 240-8501, Japan. He is now with NTT Advanced Technology Corporation, Tokyo 163-0431, Japan.

M. Ogata was with the Department of Electrical and Computer Engineering, Graduate School of Engineering, Yokohama National University, Yokohama 240-8501, Japan. He is now with Nomura Research Institute, Ltd., Yokohama 240-0003, Japan.

S. Suzuki was with the Department of Electrical and Computer Engineering, Graduate School of Engineering, Yokohama National University, Yokohama 240-8501, Japan. He is now with Ricoh Co., Ltd., Yokohama 224-0035, Japan. Digital Object Identifier 10.1109/JSTQE.2004.841720

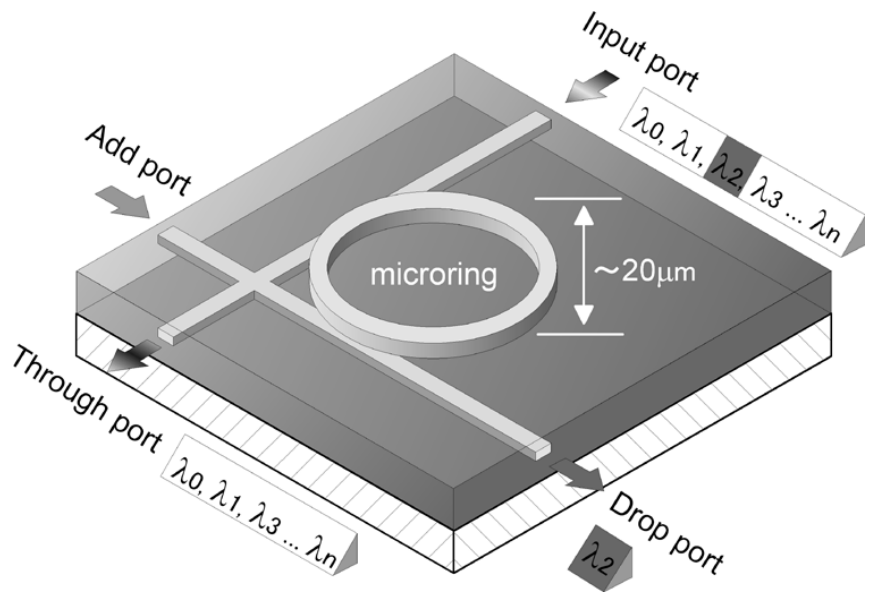

Fig. 1. VCMRR.

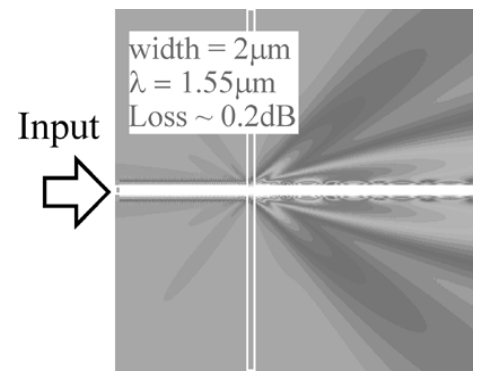

Fig. 2. Two-dimensional FDTD simulation of light scattering at the crossing point of busline waveguides.

owing to the vertically coupled configuration [5], [6], where a microring resonator with a $10-20-\mu \mathrm{m}$ radius is stacked on the crossing point of cross-grid busline waveguides [6], as shown in Fig. 1. Due to the stacked configuration, the upper and lower waveguides play different roles, i.e., the lower buried channel waveguides serve as input/output busline waveguides while the microring resonator functions as the frequency-selective element. In addition, the coupling strength between ring and busline waveguides can be controlled more precisely than the lateral coupling, because the vertical separation is obtained by the well-controlled deposition, rather than etching fine gaps. This leads to the demonstration of high- $Q$ microring resonator filters with a very small radius of 10-20 $\mu \mathrm{m}$, which exhibit a bandwidth of $0.2-1.0 \mathrm{~nm}$ and a free spectral range (FSR) of 10-25 nm.

In this VCMRR, however, the scattering loss at the crossing point is as large as $0.2 \mathrm{~dB} /$ crossing according to the finite-difference time-domain (FDTD) analysis shown in Fig. 2, although 


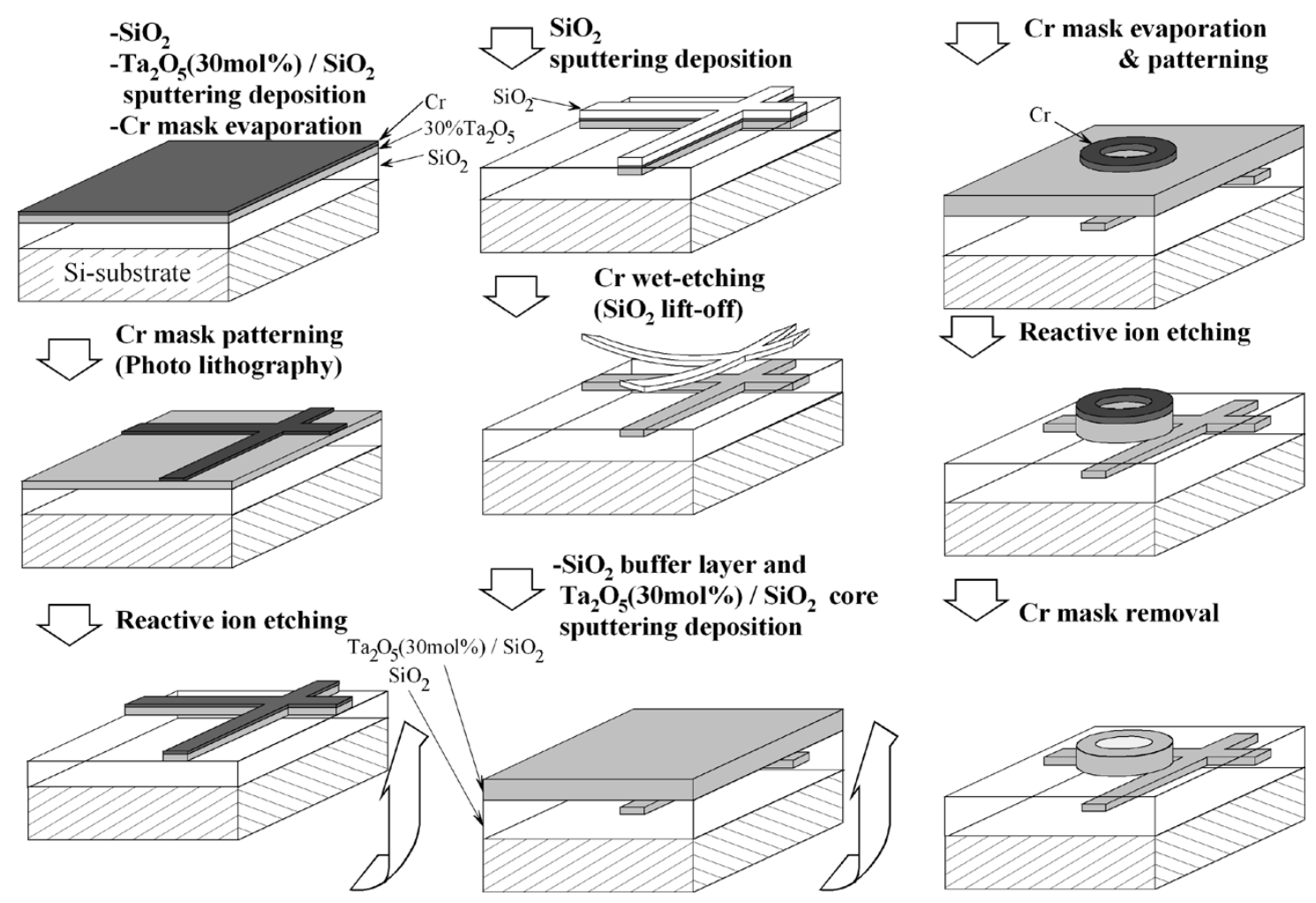

Fig. 3. Conventional fabrication process of single VCMRR filter.

the crosstalk of the crossed port is as small as $-31 \mathrm{~dB}$ [6]. This large scattering is attributed to the small spot size of the busline waveguide $(0.55 \mu \mathrm{m} \times 0.95 \mu \mathrm{m})$ resulting from the small core size (thickness $=0.5 \mu \mathrm{m}$, width $=2.0 \mu \mathrm{m}$ ). This scattering loss limits the integration scale to less than 100.

Therefore, in this study, we have introduced the multilevel crossing structure of a busline waveguide to eliminate the scattering loss. To realize this structure, the top surfaces of the buried busline and microring waveguides must be perfectly planarized. However, the top surface fabricated by the conventional liftoff process has required a step height of approximately $0.2 \mu \mathrm{m}$. Thus, we developed a new perfect planarization process by incorporating a spin-on-glass (SOG) method into the conventional liftoff process. Using this technique, a VCMRR filter with multilevel crossing busline waveguides was fabricated and clear filter responses were demonstrated by single- and high-order-series coupled microring resonators.

\section{FABRicATION PROCESS FOR MUlTileVEl INTEGRATION AND ULTRACOMPACT MICRORING RESONATOR}

\section{A. Conventional Fabrication Process}

Fig. 3 shows the fabrication process of a single VCMRR. The waveguide core layers are made of $\mathrm{Ta}_{2} \mathrm{O}_{5} / \mathrm{SiO}_{2}$ compound glass $\left(\mathrm{Ta}_{2} \mathrm{O}_{5} 30 \mathrm{~mol} \%, n=1.785 @ \lambda=1550 \mathrm{~nm}\right)$, and the cladding and separation layers are $\mathrm{SiO}_{2}(n=1.451 @ \lambda=$ $1550 \mathrm{~nm}$ ). The cladding of the microring resonator is air, and so the index contrast is $34 \%$. All the layers were deposited by an RF sputtering technique and the core was patterned by photolithography and reactive ion etching (RIE). The common
TABLE I

PROCESS CONDITION OF SPUTTERING DEPOSITION

\begin{tabular}{ll}
\hline \hline Target size & $15.24 \mathrm{~mm}$ \\
\hline Substrate size & $7.62 \mathrm{~mm}$ \\
\hline Substrate temperature & $300^{\circ} \mathrm{C}$ \\
\hline RF frequency & $13.56 \mathrm{MHz}$ \\
\hline RF power & $1.0 \mathrm{~kW}$ (Magnetron) \\
\hline RF power for substrate bias & $115 \mathrm{~W}$ (for $\mathrm{SiO}_{2}$ with substrate bias) \\
\hline Gas flow rate & $\mathrm{Ar}=5 \mathrm{sccm}, \mathrm{O}_{2}=15 \mathrm{sccm}$ \\
\hline Gas pressure & $0.67 \mathrm{~Pa}$ \\
\hline Deposition rate & $1.03 \mu \mathrm{m} / \mathrm{h}$ for $\mathrm{Ta}_{2} \mathrm{O}_{5}-\mathrm{SiO}_{2}$ (non-bias) \\
& $1.00 \mu \mathrm{m} / \mathrm{h}$ for $\mathrm{SiO}_{2}$ (non-bias) \\
& $0.85 \mu \mathrm{m} / \mathrm{h}$ for $\mathrm{SiO}_{2}$ (bias sputtering) \\
\hline \hline
\end{tabular}

TABLE II

PRocess CONDITION OF RIE

\begin{tabular}{ll}
\hline \hline Substrate size & $7.62 \mathrm{~mm}$ \\
\hline Substrate temperature & Water cooling \\
\hline RF frequency & $13.56 \mathrm{MHz}$ \\
\hline RF power & $50 \mathrm{~W}$ \\
\hline Gas flow rate & $\mathrm{C}_{2} \mathrm{~F}_{6}=15 \mathrm{sccm}$ for $\mathrm{Ta}_{2} \mathrm{O}_{5}-\mathrm{SiO}_{2}$ and \\
& $\mathrm{SiO}_{2}$ \\
\hline Gas pressure & $0.5 \mathrm{~Pa}$ \\
\hline Etching rate & $1.0 \mu \mathrm{m} / \mathrm{h}$ for $\mathrm{Ta}_{2} \mathrm{O}_{5}-\mathrm{SiO}_{2}$ and $\mathrm{SiO}_{2}$ \\
\hline \hline
\end{tabular}

process conditions of sputtering deposition and RIE are summarized in Tables I and II, respectively.

The key point of this process is to obtain busline waveguide with a flattop surface. To achieve this, we developed a liftoff process, as shown in Fig. 3. In this liftoff process, first the lower busline waveguide core is formed by RIE using the Cr mask, and the ridge-shaped core is buried by $\mathrm{SiO}_{2}$ using a bias-sputtering deposition technique. The $\mathrm{SiO}_{2}$ layer on the core is eliminated 


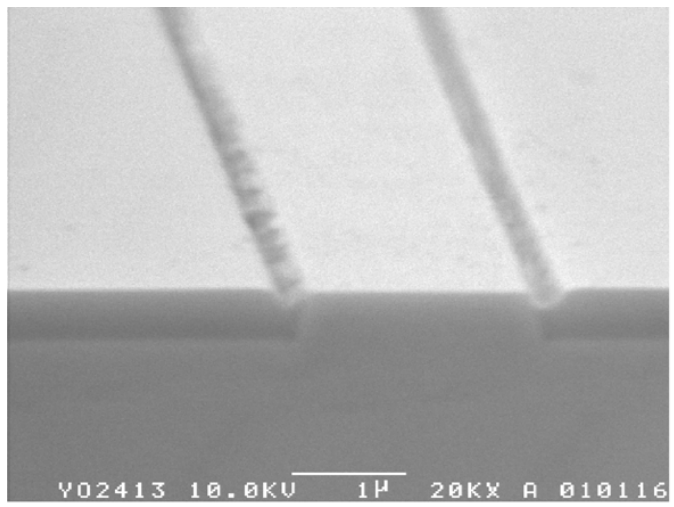

(a)

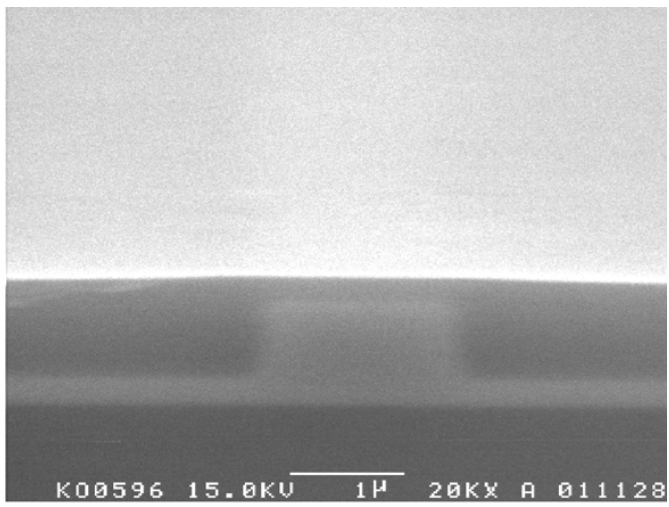

(b)

Fig. 4. Scanning electron microscope (SEM) cross-sectional photographs of busline waveguide. (a) After liftoff of Cr mask. (b) After spin coating of SOG.

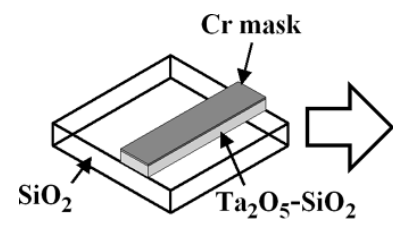

Patterning of lower busline

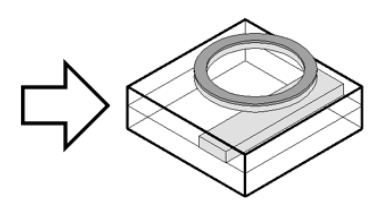

Patterning of microring resonator

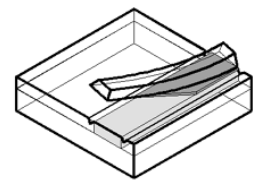

Lift-off of $\mathrm{SiO}_{2}$ cladding layer
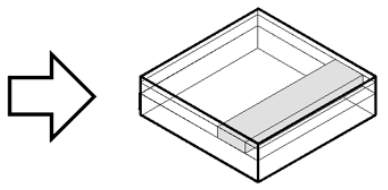

Planarization by spin coating of $\mathrm{SOG}$

Fig. 5. Improved fabrication process for multilevel VCMRR filter.

by the liftoff process by wet chemical etching the Cr mask using HY solution (made by Wako Pure Chemical Industries. Ltd., Osaka, Japan, composition is Cerium (IV) diammonium nitrate $13.3 \mathrm{wt} \%$, perchloric acid $70 \%$, and the remainder is water). Due to this liftoff process, the bump height of the top of the busline waveguide was reduced to less than $0.1 \mu \mathrm{m}$, as shown in Fig. 4(a).

\section{B. Improved Fabrication Process for Planarization}

However, this small step height should be perfectly planarized to reduce the scattering loss in the ring resonator. In particular, the perfectly planarized top surface is required for the multilevel stacking of the waveguides. Thus, we developed an improved fabrication process using a spin-on glass (SOG), as shown in Fig. 5, to planarize the top surface of the buried waveguide perfectly. The SOG is liquid before baking, and its thin film (approximately $0.2 \mu \mathrm{m}$ ) can be formed by spin coating and baking. Due to the characteristics of spin coating, a small step with a height of less than $0.1 \mu \mathrm{m}$ can be perfectly planarized, as shown in Fig. 4(b). We used the product no. OCD T1 Si-5900 made by Tokyo Ohka Kogyo Co., Ltd., as the source material of SOG. The composition is ethyl-silicate-polymer 9\% ( $\mathrm{Si} \mathrm{5.9 \% ),}$
TABLE III

PROCESS CONDITION OF SOG

\begin{tabular}{lll}
\hline \hline \multirow{2}{*}{ Spin coating } & $1 \mathrm{st}$ & $700 \mathrm{rpm} \times 5 \mathrm{sec}$ \\
\cline { 2 - 3 } & 2nd & $2000 \mathrm{rpm} \times 30 \mathrm{sec}$ \\
\hline Baking & $400^{\circ} \mathrm{C} \times 40 \mathrm{~min}$, in $\mathrm{N}_{2}$ gas \\
\hline Film thickness & $0.2 \mu \mathrm{m}$ \\
\hline \hline
\end{tabular}

ethanol $73 \%$, and methyl acetate $18 \%$. The process data are summarized in Table III.

\section{Fine Fabrication Process of Smooth Sidewall}

Another key issue is to obtain a vertical and smooth sidewall for the microring resonator. This is very important for reducing the scattering loss of the ring waveguide. Because the index contrast of the ring waveguide is extremely high $(\Delta>30 \%)$ and the ring radius is very small $(\sim 10 \mu \mathrm{m})$, the field profile of the guided mode in the microring is localized around the outer sidewall of the microring. Therefore, the scattering loss markedly increases with the roughness of sidewall.

However, it is seen from Fig. 6(b) that some appreciable amount of roughness remains on the sidewall of the microring waveguide. By careful observation of the sidewall after each fabrication process, it was found that this roughness appears 


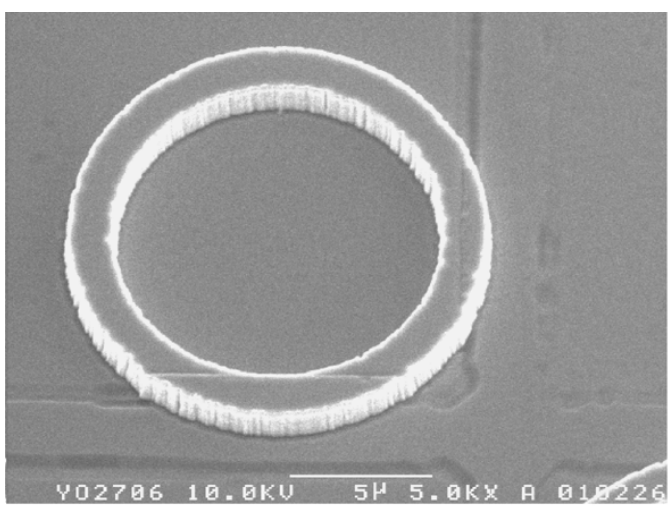

(a)

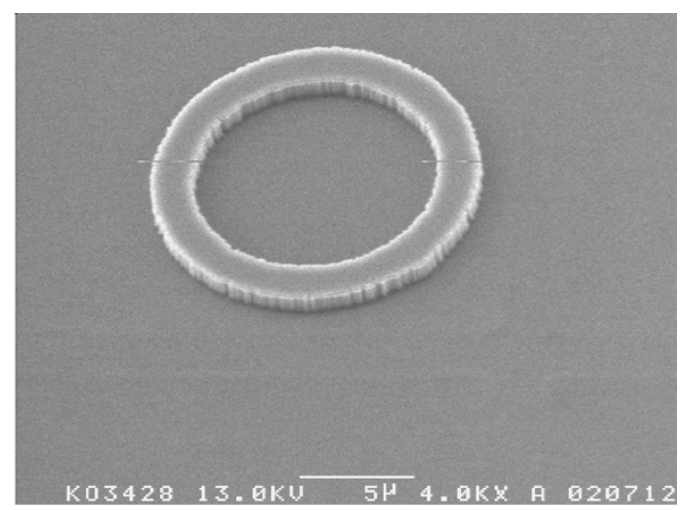

(b)

Fig. 6. SEM bird's-eye-view photographs of VCMRR with air cladding. (a) By conventional liftoff process. (b) By improved fabrication process.

TABLE IV

Process Condition of ReACtive ION ETChing $C_{r}$

\begin{tabular}{ll}
\hline \hline Substrate size & $7.62 \mathrm{~mm}$ \\
\hline Substrate temperature & Water cooling \\
\hline RF frequency & $13.56 \mathrm{MHz}$ \\
\hline RF power & $25 \mathrm{~W}$ \\
\hline Gas flow rate & $\mathrm{O}_{2}=50 \mathrm{sccm}$ \\
\hline Gas pressure & $26.6 \mathrm{~Pa}$ \\
\hline Etching rate & $0.08 \mu \mathrm{m} / \mathrm{h}$ \\
\hline
\end{tabular}

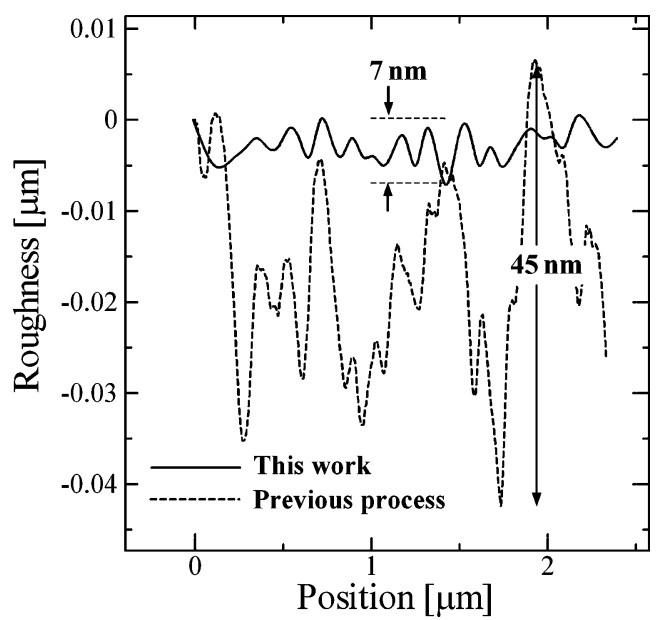

Fig. 7. Comparison of surface roughness of sidewall fabricated by conventional fabrication process and fine fabrication process of smooth sidewall.

at the patterning of the $\mathrm{Cr}$ mask using wet chemical etching. Thus, we changed the patterning process of the $\mathrm{Cr}$ mask from wet chemical etching to RIE using $\mathrm{O}_{2}$ gas and a silicone-based positive photoresist. The thickness of $\mathrm{Cr}$ mask was $100 \mathrm{~nm}$ and the condition of RIE is listed in Table IV. As a result, the roughness of the sidewall was extremely reduced from 45 to $7 \mathrm{~nm}$, as shown in Fig. 7.

The roughness shown in Fig. 7 was measured by preparing some straight waveguide patterns of which the direction is slightly slanted against the crystal orientation of the Si substrate, and a cross section of the $\mathrm{Si}$ substrate was prepared by the cleavage, so that the sidewall of the waveguide just appears on some parts of the cleaved surface. The roughness

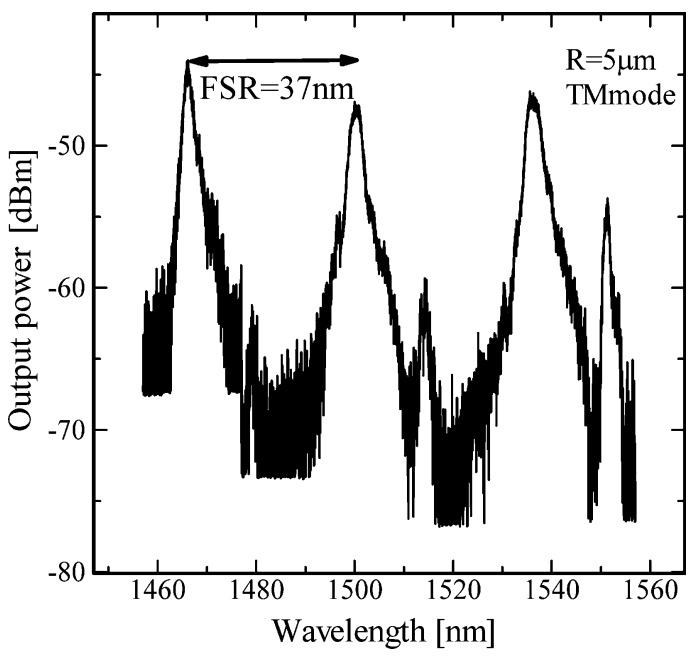

Fig. 8. Measured drop-port response of VCMRR with ring radius of $5 \mu \mathrm{m}$ produced by fine fabrication process of smooth sidewall.

was measured using a scanning electron microscope (SEM) equipped with a roughness measurement option.

\section{FABrication of VCMRR With Multilevel Crossing BUSLINE WAVEGUIDE AND ULTRACOMPACT RING RADIUS}

\section{A. Ultracompact Ring Radius and Wide FSR}

Using the fabrication process described in Section II-C, we fabricated a microring resonator (shown in Fig. 1) with a ring radius of $5 \mu \mathrm{m}$. The core material of the microring and busline waveguides is $\mathrm{Ta}_{2} \mathrm{O}_{5} / \mathrm{SiO}_{2}$ compound glass $\left(\mathrm{Ta}_{2} \mathrm{O}_{5} 30 \mathrm{~mol} \%\right.$, $n=1.785 @ \lambda=1550 \mathrm{~nm}$ ), and the cladding and separation layers are $\mathrm{SiO}_{2}(n=1.451 @ \lambda=1550 \mathrm{~nm})$. The width and thickness of the ring core were 1.2 and $0.7 \mu \mathrm{m}$, respectively. The thickness of the separation layer between the microring and busline waveguides was $0.6 \mu \mathrm{m}$. The filter response was measured using a tunable laser and an optical spectrum analyzer.

The measured drop-port response is shown in Fig. 8. An extremely large FSR of $37 \mathrm{~nm}$ was obtained. To our knowledge, this FSR is the largest value for a single-ring resonator reported thus far. If a semiconductor waveguide is used as the ring core [4], [7], [8], the optical path length in the resonator with the same $5-\mu \mathrm{m}$ radius is much longer than that of a microring made 
of dielectric material with an index of approximately 2.0, and the FSR is limited to approximately $20 \mathrm{~nm}$.

The fiber-to-fiber insertion loss of the device shown in Fig. 8 was about $45 \mathrm{~dB}$. In addition, the through-port response was not observed. This large loss is attributed to the input/output coupling loss, and the coupling imbalance, i.e., the difference of coupling efficiencies from the input busline waveguide to the ring and from the ring to the output busline waveguide. The former loss (input/output coupling loss) is due to the spot size mismatch between the busline waveguide $(0.55 \mu \mathrm{m} \times 0.95 \mu \mathrm{m})$ and the input/output fibers (about $10 \mu \mathrm{m}$ ) and is evaluated to be about $30 \mathrm{~dB}$. This coupling loss can be eliminated by incorporating the spot size transformer into the input/output ends. The latter loss is caused by the small coupling efficiency resulting from the small ring radius. Since the ring radius is so small, the effective coupling length is also smaller than those of microrings with the ring radius larger than $10 \mu \mathrm{m}$. When the coupling efficiency is small, the coupling from the microring to the output busline waveguide is very sensitive to the coupling imbalance. Therefore, when a small coupling imbalance between the input side (through-port side) and the output side (drop-port side) occurs, only a small part of the input power is transmitted to the drop port, and so the resonance drop in the through port is not observed.

To compare the filtering characteristics, we produced microring resonators, as shown in Fig. 1, with a ring radius of $20 \mu \mathrm{m}$ by the conventional process and by the fine fabrication process for a smooth sidewall. The materials and the dimension are the same as those of the device with a ring radius of $5 \mu \mathrm{m}$ except for the ring radius. The measured filter responses from the drop port and through port are shown in Fig. 9(a) and (b), respectively. The $-3-\mathrm{dB}$ bandwidth of drop-port response was improved from 0.3 to $0.2 \mathrm{~nm}$, and the rejection depth of through-port response was significantly improved from -4 to $-19 \mathrm{~dB}$. The FSRs were $10.6 \mathrm{~nm}$ for the device fabricated by the previous process and $8.9 \mathrm{~nm}$ for that by the new process. Although the ring radius was the same, the FSR was different for these devices. This seems to be caused by the difference of waveguide width of ring waveguide resulting from the fabrication error.

From these measured data, the propagation loss in the microring and the power coupling efficiency were evaluated using the formulas given in [9] (see the Appendix). The propagation loss was markedly improved from 0.47 to $0.066 \mathrm{~dB} /$ turn. The power coupling efficiencies were $3.1 \%$ for the device fabricated by the previous process and $6.1 \%$ for that by the new process.

\section{B. VCMRR With Multilevel Busline Waveguide}

Using the new fabrication process for planarization described in Section II-B, a single-ring resonator with a multilevel busline waveguide [10], as shown in Fig. 10, was fabricated. The core and cladding materials are the same as those described in the preceeding subsection. The width and thickness of the ring core were 1.5 and $1.0 \mu \mathrm{m}$, respectively. The thickness of the separation layer was $0.5 \mu \mathrm{m}$, and the ring radius was $25 \mu \mathrm{m}$. Since the microring core is buried by the $\mathrm{SiO}_{2}$ cladding, the core width of $1.5 \mu \mathrm{m}$ satisfies the single-mode condition.

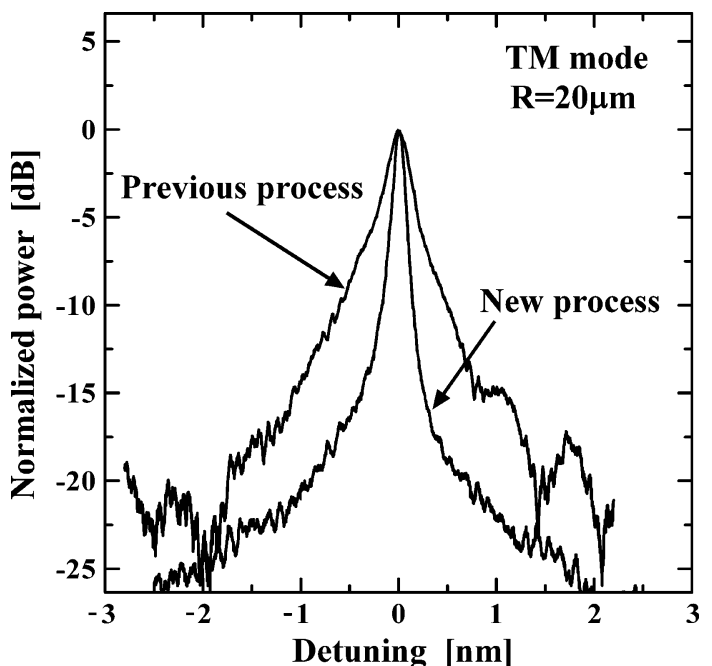

(a)

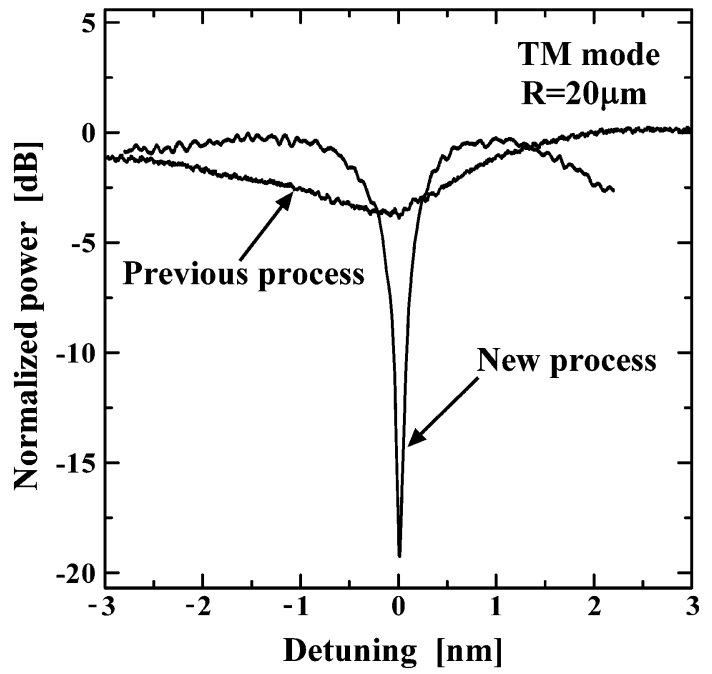

(b)

Fig. 9. Comparison of filter responses of VCMRR produced by conventional fabrication process and fine fabrication process of smooth sidewall. (The resolution was $0.01 \mathrm{~nm}$.) (a) Drop-port responses. (b) Through-port responses.

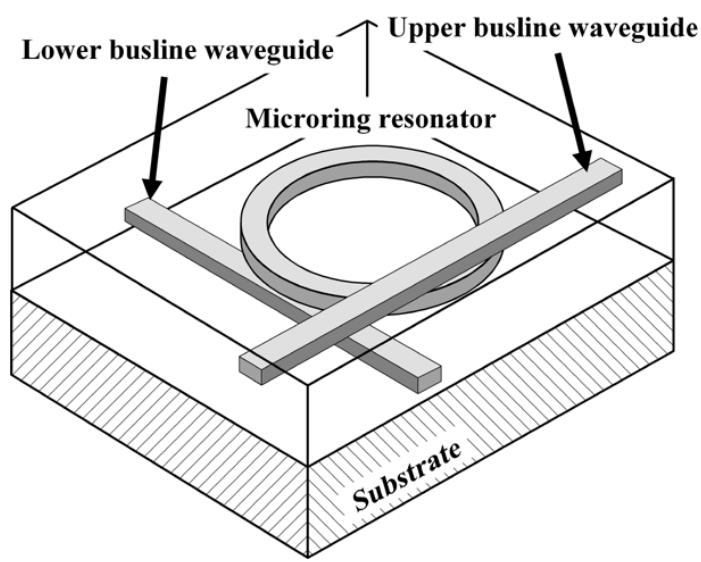

Fig. 10. VCMRR filter with multilevel crossing busline waveguide.

The filter response was measured using a tunable laser and an optical spectrum analyzer. The results for the through port and drop port are shown in Fig. 11. Clear filter responses with 


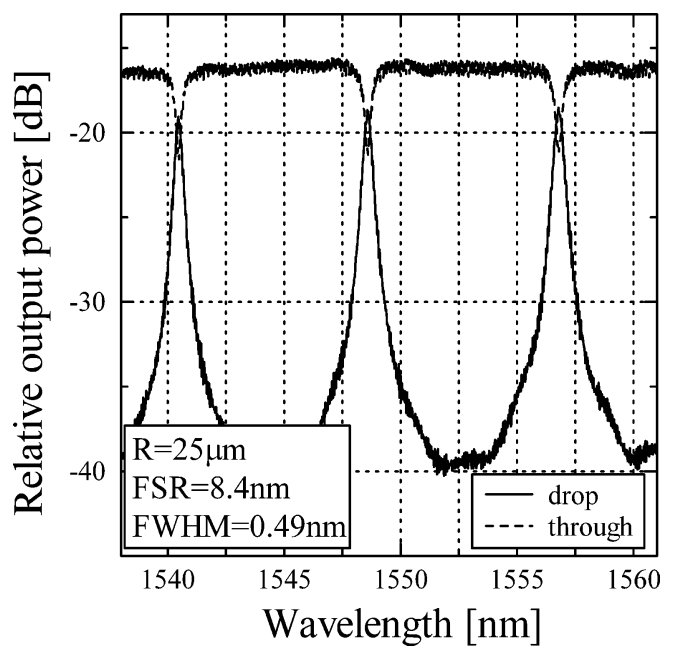

Fig. 11. Measured filter response of single-microring resonator filter with multilevel crossing busline waveguide (TE mode).

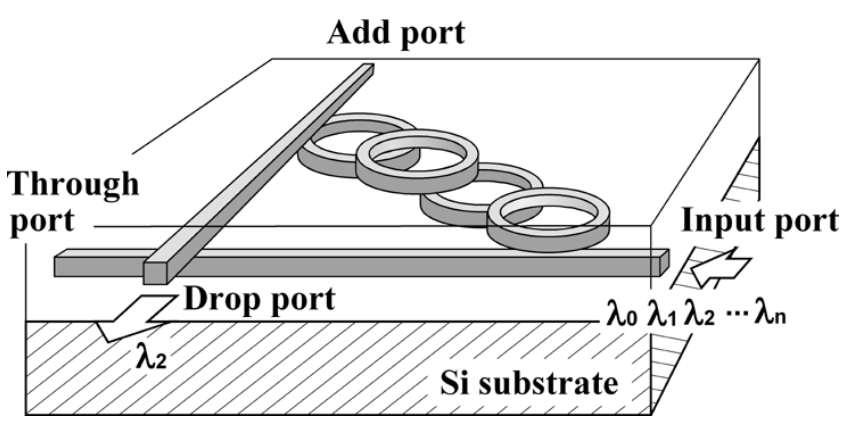

Fig. 12. Quadruple series-coupled microring resonator filter with multilevel crossing busline waveguide.

an FSR of $8.4 \mathrm{~nm}$ and a full-width at half-maximum (FWHM) bandwidth of $0.23 \mathrm{~nm}$ were observed.

A series-coupled quadruple-microring resonator with multilevel crossing busline waveguides, as shown in Fig. 12, was also fabricated by the same process. In contrast to the single-ring device shown in Fig. 10, this structure consists of two core layers, i.e., both layers involve the busline waveguide and the microring resonator and those waveguides are coupled to each other in the vertical direction. Therefore, all the couplings between the busline and the microring and between the microrings can be precisely controlled by adjusting vertical distance, i.e., the thickness of the separation layer. In addition, the busline waveguides are also crossed in different layers, and so the scattering at the crossing point will not occur. Due to the quadruple series-coupled configuration, a boxlike filter response [11], [12] is expected.

The measured filter response of the quadruple series-coupled microring resonator with a ring radius of $60 \mu \mathrm{m}$ is shown in Fig. 13. The core and cladding materials are the same as those described in the preceeding subsection. The width and thickness of the ring core were 1.4 and $0.5 \mu \mathrm{m}$, respectively. The thickness of the separation layer was $0.2 \mu \mathrm{m}$.

A clear boxlike filter response with an FWHM bandwidth of $1.12 \mathrm{~nm}$ and an FSR of $3.5 \mathrm{~nm}$ was observed. A small ripple of approximately $1.5 \mathrm{~dB}$ was observed in the passband. This seems to be caused by the strong coupling between microrings and also

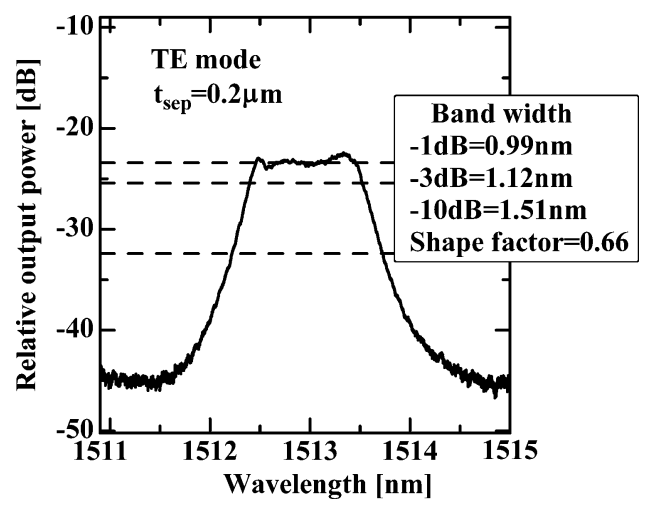

Fig. 13. Measured filter response of quadruple series-coupled microring resonator filter with multilevel crossing busline waveguide.

by the small difference in resonant wavelength between four microrings, which results from the fabrication error, and can be reduced by improving fabrication accuracy. The shape factor which is defined by the ratio of $-1-\mathrm{dB}$ bandwidth to $-10-\mathrm{dB}$ bandwidth was evaluated to be 0.66 , which is close to the theoretical value of 0.68 for the quadruple series-coupled ring resonator that is maximally flat.

\section{CONCLUSION}

To achieve the multilevel integration of busline waveguides and microring resonators, a new fabrication technology to planarize perfectly the top surface of each buried waveguide and a fine etching method to fabricate microring waveguides with very smooth sidewalls were developed. An ultracompact microring resonator with an FSR of $37 \mathrm{~nm}$ was successfully demonstrated by the latter method, and a clear and boxlike filter response was obtained by a quadruple series-coupled microring with a multilevel integrated structure of busline and microring waveguides. From these results, the effectiveness of these fine fabrication technologies was verified.

\section{APPENDIX}

Let us assume a ring resonator with radius $R$ coupled to a busline waveguide and add-drop waveguide. When the propagation constants in the ring resonator and the busline and add-drop waveguides are all the same, the coupling efficiency of the optical power in the coupling region can be expressed by $K$ to the through port and $(1-K)$ to the drop port. Thus, $K$ corresponds to the power fraction coupled from the busline waveguide to the ring waveguide, and $(1-K)$ corresponds to that which passes straight through the busline waveguide. In this case, the coupling efficiency $K$ and the propagation loss in the ring resonator $\alpha$ can be obtained by the following formulas [9]:

$$
\begin{aligned}
\alpha & =\frac{2 B \sqrt{D}}{\pi R\left(\frac{2 \mathrm{FSR}}{\pi}+B\right)} \quad[\mathrm{Np} / \mathrm{m}] \\
K & =\frac{2 B(1-\sqrt{D})}{\frac{2 \mathrm{FSR}}{\pi}+B}
\end{aligned}
$$

where $B$ is the $-3-\mathrm{dB}$ bandwidth in the drop-port response and $D$ is the depth of resonance in the through-port response. Thus, 
$\alpha$ and $K$ can be obtained from the measured data of drop-port and through-port responses.

\section{REFERENCES}

[1] S. Suzuki, K. Shuto, and Y. Hibino, "Integrated optic ring resonators with two stacked layers of silica waveguides on Si," IEEE Photon. Technol. Lett., vol. 4, no. 11, pp. 1256-1258, Nov. 1992.

[2] B. E. Little, J. S. Foresi, G. Steinmeyer, E. R. Thoen, S. T. Chu, H. A. Haus, E. P. Ippen, L. C. Kimerling, and W. Greene, "Ultra-compact $\mathrm{Si} / \mathrm{SiO} 2$ microring resonator optical channel dropping filters," IEEE Photon. Technol. Lett., vol. 10, no. 4, pp. 549-551, Oct. 1998.

[3] C. K. Madsen, "Efficient architectures for exactly realizing optical filters with optimum band-pass designs," IEEE Photon. Technol. Lett., vol. 10, no. 8, pp. 1136-1138, Aug. 1998.

[4] D. Rafizadeh, J. P. Zhang, S. C. Hagness, A. Taflov, K. A. Stair, S. T. Ho, and R. C. Tiverio, "Waveguide-coupled AlGaAs/GaAs microcavity ring and disk resonators with high finesse and $21.6 \mathrm{~nm}$ free spectral range," Opt. Lett., vol. 22, no. 16, pp. 1244-1246, Aug. 1997.

[5] B. E. Little, S. T. Chu, W. Pan, D. Ripin, T. Kaneko, Y. Kokubun, and E. Ippen, "Vertically coupled glass microring resonator channel dropping filters," IEEE Photon. Technol. Lett., vol. 11, no. 2, pp. 215-217, Feb. 1999.

[6] S. T. Chu, B. E. Little, W. Pan, T. Kaneko, S. Sato, and Y. Kokubun, "An eight-channel add-drop filter using vertically coupled microring resonators over a cross grid," IEEE Photon. Technol. Lett., vol. 11, no. 6, pp. 691-693, Jun. 1999.

[7] M. K. Chin, C. Youtsey, W. Zhao, T. Pierson, Z. Ren, S. L. Wu, L. Wang, Y. G. Zhao, and S. T. Ho, "GaAs microcavity channel-dropping filter based on a race-track resonator," IEEE Photon. Technol. Lett., vol. 11, no. 12, pp. 1620-1622, Dec. 1999.

[8] D. V. Tishinin, P. D. Dapkus, A. E. Bond, I. Kim, C. K. Lin, and J. O'Brien, "Vertical resonant couplers with precise coupling efficiency control fabricated by wafer bonding," IEEE Photon. Technol. Lett., vol. 11, no. 8, pp. 1003-1005, Aug. 1999.

[9] T. Ito and Y. Kokubun, "Nondestructive measurement of loss and coupling efficiency in microring resonator filters from filter responses," Jpn. J. Appl. Phys., vol. 43, no. 3, pp. 1002-1005, Mar. 2004.

[10] Y. Hatakeyama, T. Hanai, S. Suzuki, and Y. Kokubun, "Loss-less multilevel crossing of busline waveguide in vertically coupled microring resonator filter," IEEE Photon. Technol. Lett., vol. 16, no. 2, pp. 473-475, Feb. 2004.

[11] J. V. Hryniewicz, P. P. Absil, B. E. Little, R. A. Wilson, and P.-T. Ho, "Higher order filter response in coupled microring resonators," IEEE Photon. Technol. Lett., vol. 12, no. 3, pp. 320-322, Mar. 2000.

[12] Y. Yanagase, S. Suzuki, Y. Kokubun, and S. T. Chu, "Vertical triple series-coupled microring resonator filter for passband flattening and expansion of free spectral range," Jpn. J. Appl. Phys., vol. 41, no. 2A, pp. L141-L143, Feb. 2002.

Yasuo Kokubun (M'85) was born in Fukushima, Japan, on July 7, 1952. He received the B.E. degree from Yokohama National University, Yokohama, Japan, in 1975 and the M.E. and Dr.Eng. degrees from the Tokyo Institute of Technology, Tokyo, Japan, in 1977 and 1980, respectively.

After he worked for the Research Laboratory of Precision Machinery and Electronics, Tokyo Institute of Technology, as a Research Associate from 1980 to 1983 , he joined Yokohama National University as an Associate Professor in 1983 and is now a Professor in the Department of Electrical and Computer Engineering. From 1984 to 1985, he was also with AT\&T Bell Laboratories, Holmdel, NJ, as a Visiting Reseacher and was engaged in research on a novel waveguide on a semiconductor substrate (ARROW) for integrated optics. From 1996 to 1999, he was the Project Leader of the three-dimensional microphotonics project at the Kanagawa Academy of Science and Technology. His current research is in integrated photonics, particularly waveguide-type functional devices and three-dimensional integrated photonics.

Prof. Kokubun is a Member of the Japan Society of Applied Physics and the Optical Society of America.
Yutaka Hatakeyama was born in Tokyo, Japan on February 20, 1979. He received B.E. and M.E degrees in electrical and computer engineering from Yokohama National University, Yokohama, Japan, in 2001 and 2003, respectively.

He is now with NTT Advanced Technology Corporation, Tokyo, Japan. His main research interests include optical-waveguide-type functional devices.

Mr. Hatakeyama is a member of the Japan Society of Applied Physics (JSAP).

Masashi Ogata was born in Kanagawa, Japan, on November 28, 1979. He received the B.E. and M.E degrees in electrical and computer engineering from Yokohama National University, Yokohama, Japan, in 2002 and 2004, respectively.

He is now with Nomura Research Institute, Ltd., Yokohama, Japan. His main research interests include optical-waveguide-type functional devices.

Mr. Ogata is a member of the Japan Society of Applied Physics (JSAP).

Shuichi Suzuki was born in Kanagawa, Japan, on October 6, 1976. He received the B.E., M.E and Dr.Eng. degrees in electrical and computer engineering from Yokohama National University, Yokohama, Japan, in 1999, 2001, and 2003, respectively.

He is now with Ricoh Co., Ltd., Yokohama, Japan. His main research interests include waveguide-type functional devices.

Dr. Suzuki is a member of the Institute of Electronics, Information, and Communication Engineers (IEICE) of Japan.

Nobuhiro Zaizen was born in Osaka, Japan, on April 17, 1981. He received the B.E. degree in electrical and computer engineering from Yokohama National University, Yokohama, Japan, in 2004. He is currently working toward the M.E. degree at Yokohama National University.

His main research interests include optical-waveguide-type functional devices. 